\title{
Structural, Thermal and Surface Properties of a Formulated Sunscreen: Zn/Al-nitrate Layered Double Hydroxide Intercalated with 4-aminobenzoic Acid
}

\author{
Fadhlin Sakina MOHD RAJIDI, Mazlina MUSA*, \\ Iman Nur Fathihah Abdul AZIZ and Rozita YAHAYA \\ Department of Chemistry, Faculty of Science and Mathematics, Sultan Idris Education University, \\ Perak, Malaysia
}

('Corresponding author's e-mail: mazlinam@fsmt.upsi.edu.my)

Received: 23 April 2020, Revised: 1 May 2021, Accepted: 7 May 2021

\begin{abstract}
Intercalation of 4-amino benzoic acid (4-AB) into $\mathrm{Zn} / \mathrm{Al}$-nitrate layered double hydroxide (ZAL2) to form $\mathrm{Zn} / \mathrm{Al}-4 \mathrm{AB}$ (ZALAB) compound was successfully synthesized from nitrate salt by using direct co-precipitation method. ZALAB compound was confirmed by Powder X-ray diffraction (PXRD) pattern with a basal spacing of $15.3 \AA$. The presence of $\mathrm{COO}^{-}$and $\mathrm{C}_{6} \mathrm{H}_{5} \mathrm{NH}_{2}$ functional groups of 4-AB at 1,517 and $1,178 \mathrm{~cm}^{-1}$ in Fourier transform infrared (FTIR) spectrum reinforced 4-AB intercalated into the resulting compound. Based on the thermal analysis, 4-AB in ZALAB compound was more stable compared to pure 4-AB. Breuneur, Emmet and Teller/Barret-Joyner-Halenda (BET/BJH) analyses demonstrated that ZALAB compound is a mesopores-type IV compound with $4.25 \mathrm{~m}^{2} / \mathrm{g}$ surface area. This material is an irregular shape with different sizes of particles. ZALAB is a potential sunscreen formulation that minimizes the harmful effect on the skin, improving pharmaceutical technology in the skincare treatment.
\end{abstract}

Keywords: Zn/Al layered double hydroxide, 4-aminobenzoic acid, Sunscreen formulation, Intercalation, Skin

\section{Introduction}

Prolonged exposure to UV radiations can cause harmful effects on human skin such as sunburns, skin cancers, cutaneous photoaging, and wrinkling [1]. Therefore, a sunscreen is very important to protect human skin from UV radiation. 4-AB, which is shown in Figure 1(a), is a sunscreen composed of carboxylic and aromatic groups that can potentially filter UV-B radiation. However, some studies reported that the use of 4-AB can pose sunscreen intolerances on skin, namely; photoallergic and allergic contact dermatitis $[2,3]$. Therefore, many researchers have extensively investigated the development of new sunscreen formulations by intercalating sunscreen into layered double hydroxides (LDHs). This is due to several advantages posed by LDH when sunscreen is intercalated with inorganic layers to formulate sunscreen. The advantages include stability when the organic molecules are encapsulated in inorganic lamellar solids, the absence of direct contact of sunscreen to the skin, which can minimize allergic problems to the skin, and the absorption of UV in the UV-A, UV-B and UV-C regions [4-9]. LDH (Figure 1(b)) is known as an ionic clay that is based on the brucite $\left(\mathrm{Mg}(\mathrm{OH})_{2}\right)$ structure with the general formula of $\left[\mathrm{M}^{2+}{ }_{1-\mathrm{x}} \mathrm{M}^{3+}{ }_{\mathrm{x}}(\mathrm{OH})_{2}\right]^{\mathrm{x}+}\left(\mathrm{A}^{\mathrm{n}-}\right)_{\mathrm{x} / \mathrm{n}} \cdot \mathrm{yH}_{2} \mathrm{O} \cdot \mathrm{M}^{2+}$ to $\mathrm{M}^{3+}$ cations substitution induces a positive charge to the layer and is balanced by interlayer exchangeable anions $\left(\mathrm{A}^{\mathrm{n}-}\right)$ in the interlayer domain as shown in [10]. Owing to the rich intercalation chemistry of LDHs in the sunscreen formulation, this 
present work deals with the structural, thermal, and surface studies of the intercalation of 4-AB anion into ZAL2 inorganic gallery forming ZALAB.

(a)

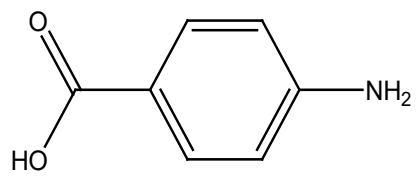

(b)

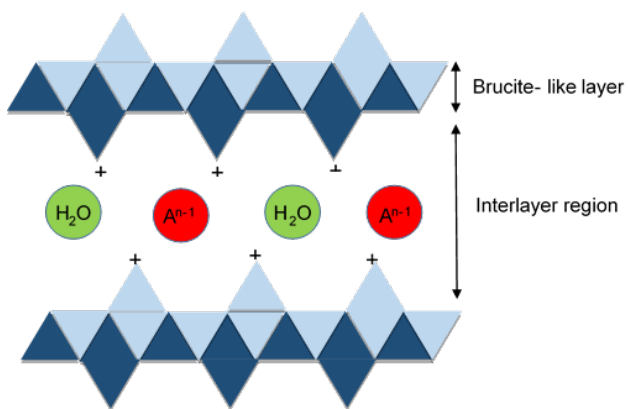

Figure $14-\mathrm{AB}$ molecular structure (a) and structure of LDH (b).

\section{Materials and methods}

\section{Synthesis of ZAL2 material}

ZAL2 was prepared at $\mathrm{pH} 7$ by using direct co-precipitation method with modification procedure [9]. Zinc nitrate hexahydrate $\left(\mathrm{Zn}\left(\mathrm{NO}_{3}\right)_{2} .6 \mathrm{H}_{2} \mathrm{O}\right)$ and aluminium nitrate nanohydrate $\left(\mathrm{Al}\left(\mathrm{NO}_{3}\right)_{3} .9 \mathrm{H}_{2} \mathrm{O}\right)$ with molar ratio 2:5 were dissolved in deionized water. The solution was titrated with sodium hydroxide $(\mathrm{NaOH})$ until a $\mathrm{pH}$ of 7 under nitrogen $\left(\mathrm{N}_{2}\right)$ atmosphere was achieved to minimize contamination by atmospheric $\mathrm{CO}_{2}$ [11]. Afterward, the slurry was aged for $18 \mathrm{~h}$ at $70{ }^{\circ} \mathrm{C}$ in the oil bath shaker. The precipitate obtained was washed with deionized water and dried overnight in an oven at $70{ }^{\circ} \mathrm{C}$. The sample was grounded into fine powder and kept in vial for further characterizations.

\section{Synthesis of ZALAB material}

ZALAB has been prepared by direct co-precipitation method following the procedure described in literature [9] with modification. Aqueous solutions of $\left.\mathrm{Zn}\left(\mathrm{NO}_{3}\right)_{2} \cdot 6 \mathrm{H}_{2} \mathrm{O}\right), \mathrm{Al}\left(\mathrm{NO}_{3}\right)_{3} \cdot 9 \mathrm{H}_{2} \mathrm{O}$ and 4-AB were mixed together with an initial molar ratio of 2:5:0.8. Then, the solution was titrated with slow addition of $2 \mathrm{M}$ sodium hydroxide $(\mathrm{NaOH})$ and under $\mathrm{N}_{2}$ atmosphere was adjusted to $\mathrm{pH} 7$. The slurry obtained was aged for $18 \mathrm{~h}$ in an oil bath shaker at $70{ }^{\circ} \mathrm{C}$. Next, the precipitate was centrifuged and thoroughly washed with deionized water. Finally, the sample was dried overnight in an oven at $70{ }^{\circ} \mathrm{C}$ and grounded into fine powder for further characterizations.

\section{Characterization}

PXRD patterns were recorded at room temperature with a Bruker AXS Germany using filtered $\mathrm{CuK}_{\alpha}$ radiation $(\lambda=1.5406 \AA)$ at $40 \mathrm{kV}$ and $40 \mathrm{~mA}$. Data was collected at $2 \theta$ from $2^{\circ}$ to $60^{\circ}$. FTIR spectra was recorded by using Nexus spectrophotometer. The measurements were analyzed in the transmission mode with an accumulation of 32 scans in the range of $400-4,000 \mathrm{~cm}^{-1}$. Thermogravimetric analyses

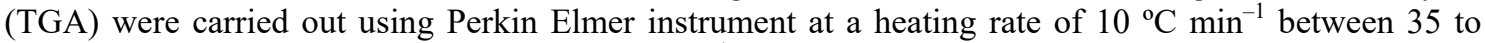
$1,000{ }^{\circ} \mathrm{C}$ under the oxygen flow of $20 \mathrm{~mL} \mathrm{~min}$. Surface area characterization of the materials was analyzed using Micromeritics, ASAP2000. On the other hand, the surface morphologies of the materials were scanned with Field Emission Scanning Electron Microscopy (FESEM) using ZEISS supra 40 VP. 


\section{Results and discussion}

Powder X-ray Diffraction (PXRD) pattern

PXRD patterns of ZAL2 and ZALAB are illustrated in Figure 2. It shows that both materials were crystallized with a well-ordered layered structure. ZAL2 synthesized with nitrate as an interlamella anion exhibited a typical diffraction peak of 003 having a basal spacing of $8.8 \AA$. After intercalation, the basal spacing of ZAL2 expanded to $15.3 \AA$ in ZALAB compound. Both basal spacing of compounds obtained were similar to the report by He et al. in 2004 [9]. The expansion of interlamella gallery was due to the inclusion of 4-AB into the inorganic layer of ZAL2, indicating that 4-AB anions have more affinity replacing $\mathrm{NO}_{3}^{-}$in the inorganic interlayer [12-14]. Figure 3(a) illustrates the three-dimensional molecular size of 4-AB, which was estimated by using Chemoffice software. The width, length, and thickness of 4$\mathrm{AB}$ wee 5.1, 8.4 and $3.5 \AA$, respectively. The proposed spatial arrangement of 4-AB within the ZAL2 interlayer region based on the basal spacing is shown in Figure 3(b). The basal spacing of ZALAB was $15.3 \AA$ obtained after taking into account $4.8 \AA$ thickness of brucite-like layer, while gallery height was $10.5 \AA[8-9,15,16]$. This is in agreement with the basal spacing obtained from PXRD pattern of ZALAB as displayed in Figure 1.

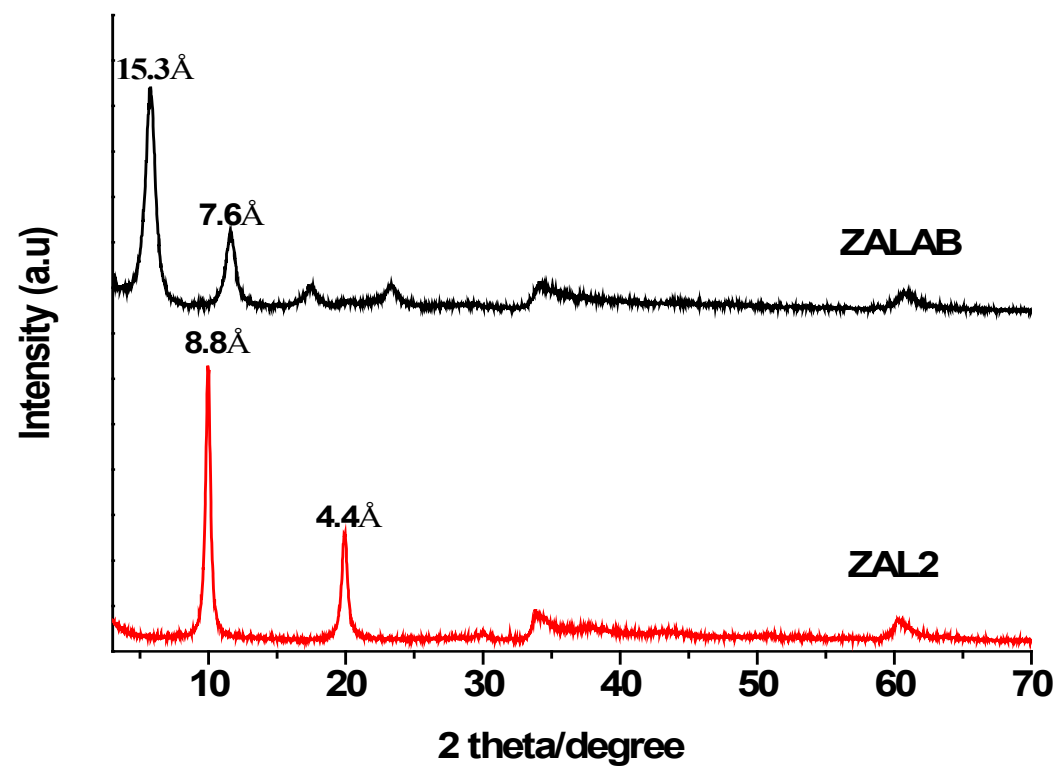

Figure 2 PXRD pattern of ZAL2 and ZALAB. 
(a)

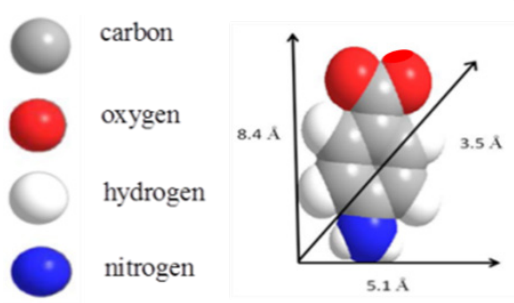

(b)

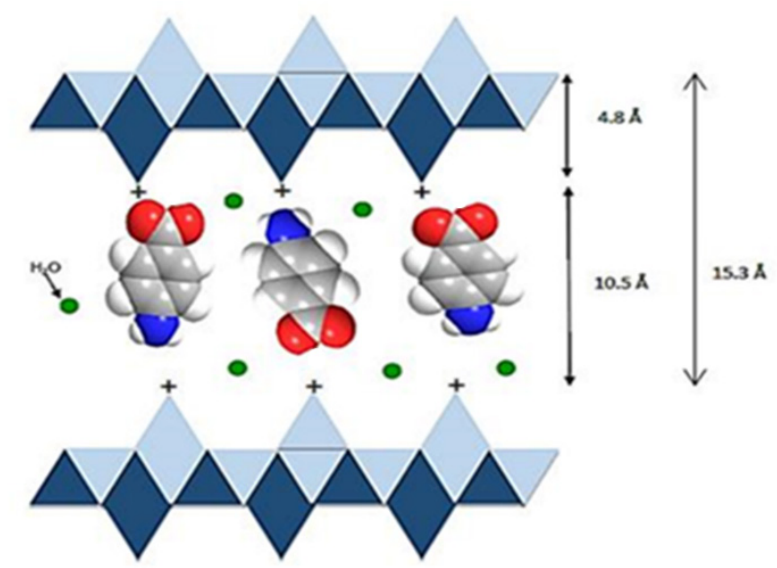

Figure 3 Three-dimensional molecular structure of (a) 4-AB and (b) proposed spatial orientation of 4-AB in ZAL2 interlayer.

\section{Fourier Transform Infrared (FTIR) spectrum}

FTIR spectra of 4-AB, ZAL2 and ZALAB nanocomposite are presented in Figure 4. The spectrum of ZALAB can be observed as a combination of 4-AB and ZAL2 vibration signals. Specifically, the presence of elongation band of $1,178 \mathrm{~cm}^{-1}$ aromatic amine $\left(\mathrm{C}_{6} \mathrm{H}_{5} \mathrm{NH}_{2}\right)$ and $1,517 \mathrm{~cm}^{-1}$ carboxylate $\left(\mathrm{COO}^{-}\right)$ groups give strong signals that $4-\mathrm{AB}$ anions were intercalated in the interlayer gallery of ZALAB. An absorption band at $1,664 \mathrm{~cm}^{-1}$ was also detected which is assigned to the stretching vibration of the 4-AB carbonyl group $(\mathrm{C}=\mathrm{O})$ in ZALAB. A broad absorption peak of the interlayers hydroxyl group and physically adsorbed water molecules could be observed at $3,427 \mathrm{~cm}^{-1}$. The $3,456 \mathrm{~cm}^{-1}$ peak is due to the $\mathrm{N}-\mathrm{H}$ bond belongs to $4-\mathrm{AB}$ disappeared in the ZALAB spectrum with the establishment of the hydroxyl group in the intercalation compound $[1,6,8]$.

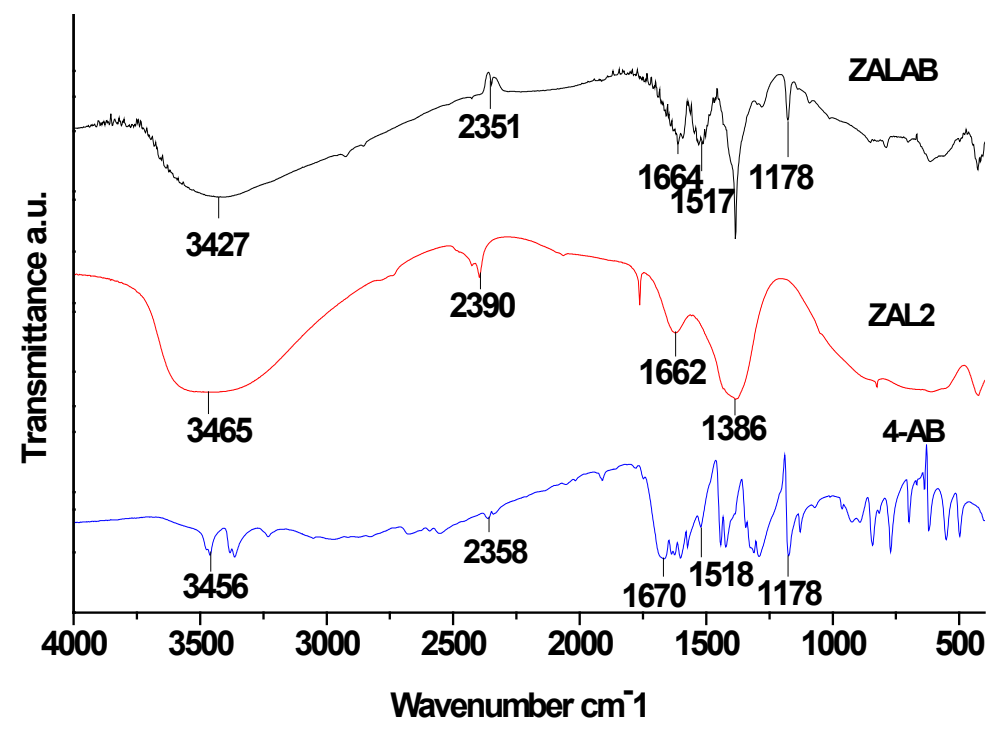

Figure 4 FTIR spectrum of 4-AB, ZAL2 and ZALAB materials. 


\section{Thermogravimetric analysis and differential thermal gravimetric (TGA/DTG)}

Figures 5 to 7 demonstrates the TGA/DTG thermograms of ZAL2, ZALAB and 4-AB. Based on the results, ZAL2 curve in Figure 5 clearly exhibited 3 decomposition stages of the compound in which the $1^{\text {st }}$ weight loss stage at $106.8^{\circ} \mathrm{C}$ was associated with the release of adsorbed and interlayers molecule of water. The second and the $3^{\text {rd }}$ steps weight loss at around 255.5 to $321.4{ }^{\circ} \mathrm{C}$ were due to the dehydroxylation of metal hydroxide layers and decomposition of nitrate anions. A tiny signal at $542.9^{\circ} \mathrm{C}$ was also detected, which corresponds to the degradation of residual adsorbed nitrate that collapsed together with dehydroxylation in the ZAL2 layer followed by the formation of metals oxides [17,18]. Figure 6 shows 4 major stages of ZALAB weight loss. The $1^{\text {st }}$ stage was $9.44 \%$ at $98{ }^{\circ} \mathrm{C}$. This was due to the elimination of physisorbed and interlayer water [19]. The second and the $3^{\text {rd }}$ stages were attributed to the organic molecules combustion of the encapsulated 4-AB in ZALAB material occurred between 262 and $437^{\circ} \mathrm{C}$. The final decomposition stage at $863^{\circ} \mathrm{C}$ was assigned to the combustion of the brucite-like layer, which was transformed into metal oxide [20]. A thermal analysis curve of ZALAB shows that the compound is more stable compared to the pure form of $4-\mathrm{AB}$ that was decomposed at $231^{\circ} \mathrm{C}$ as shown in Figure 7. The thermal stability of intercalated $4-\mathrm{AB}$ was enhanced due to confinement in LDH layers as a result of electrostatic attraction between di-anion B9 and positively charged brucite-like layers of LDH [15].

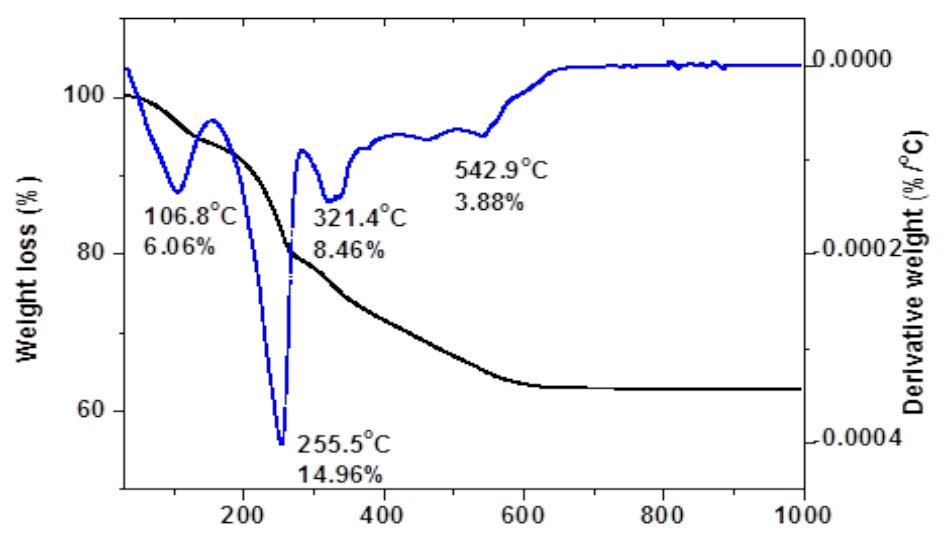

Figure 5 TGA/DTG thermogram of ZAL2.

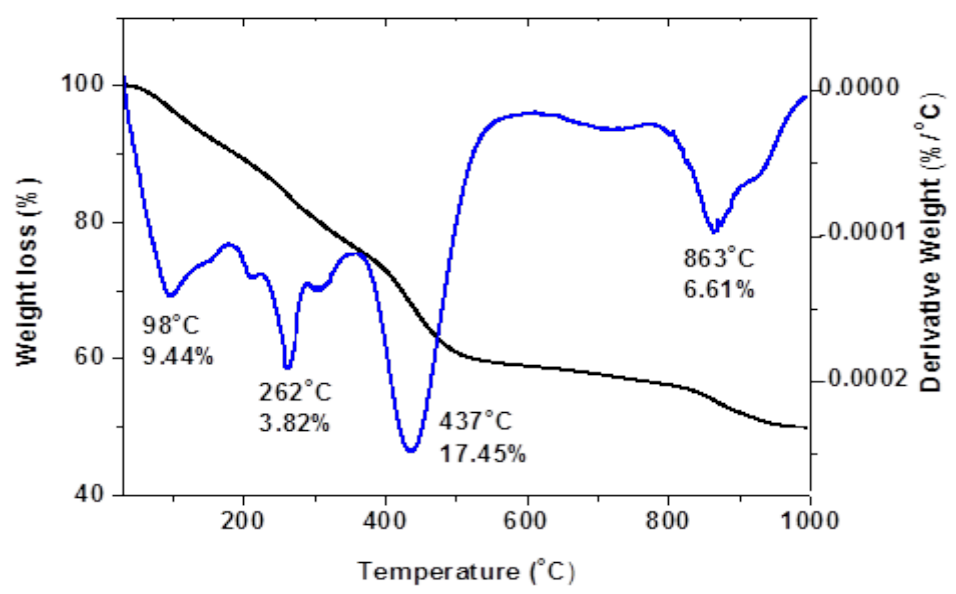

Figure 6 TGA/DTG thermogram of ZALAB. 


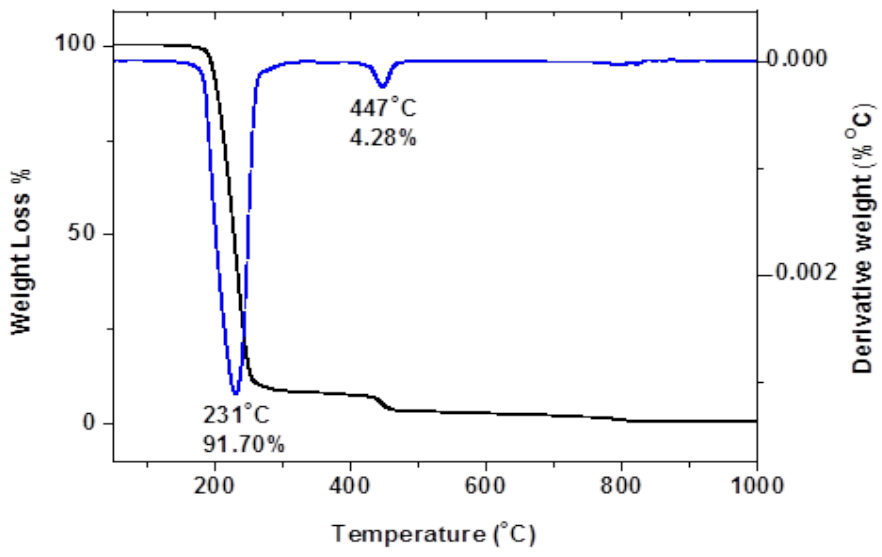

Figure 7 TGA/DTG thermogram of 4-AB.

\section{Surface area properties}

Nitrogen adsorption and desorption isotherms of ZAL2 and ZALAB are shown in Figure 8. Both materials are type IV adsorption isotherm with H3-type hysteresis loop by the International Union of Pure and Applied Chemistry (IUPAC) classification. This classification is due to the mesopores materials that are comprised of the plate-like particle with slit-shaped pores [21]. ZAL2 isotherm shows that the nitrogen adsorbate uptake gradually increases at a relative pressure range of $0.0-0.8$ and speedy uptake around $0.8-1.0$ with maximum adsorption at $0.86 \mathrm{~cm}^{3} / \mathrm{g}$. ZALAB shows rapid adsorption of nitrogen around the relative pressure range of $0.0-0.1$ and $0.8-1.0$ with maximum uptake at $0.92 \mathrm{~cm}^{3} / \mathrm{g}$. BJH pore size distribution image in Figure 9 shows that ZALAB possesses a wide range of pore distribution compared to ZAL2. In addition, the recorded BET surface area and BJH pore diameter for the intercalated compound are $4.25 \mathrm{~m}^{2} \mathrm{~g}^{-1}$ and $134.9 \AA$, respectively, as presented in Table $\mathbf{1}$. The increment of surface area and pore size from ZAL2 to ZALAB was attributed to the modification of the materials pore texture when 4-AB anions entered ZAL2 interlayer gallery to form ZALAB [13]. 


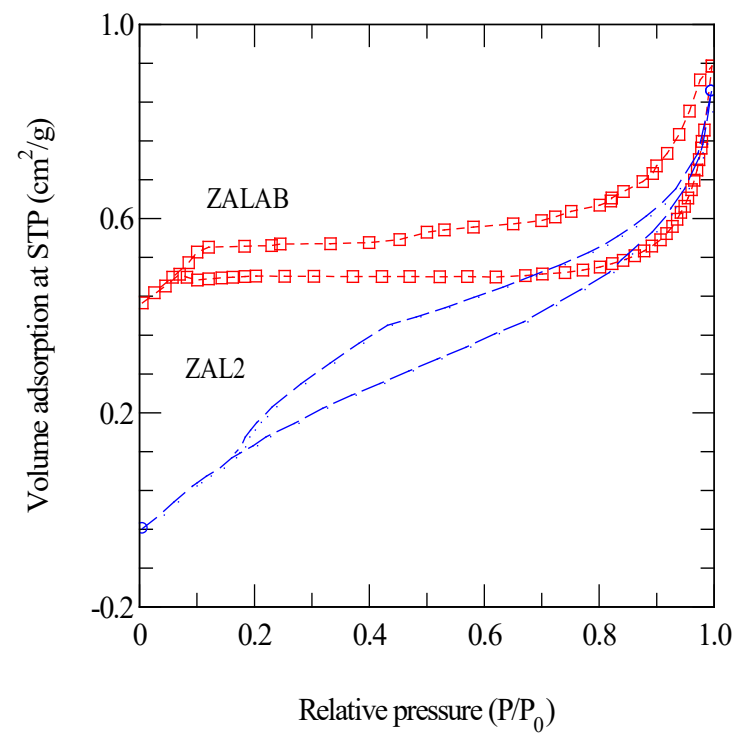

Figure 8 Nitrogen adsoprtion-desorption isotherms of ZAL2 and ZALAB.

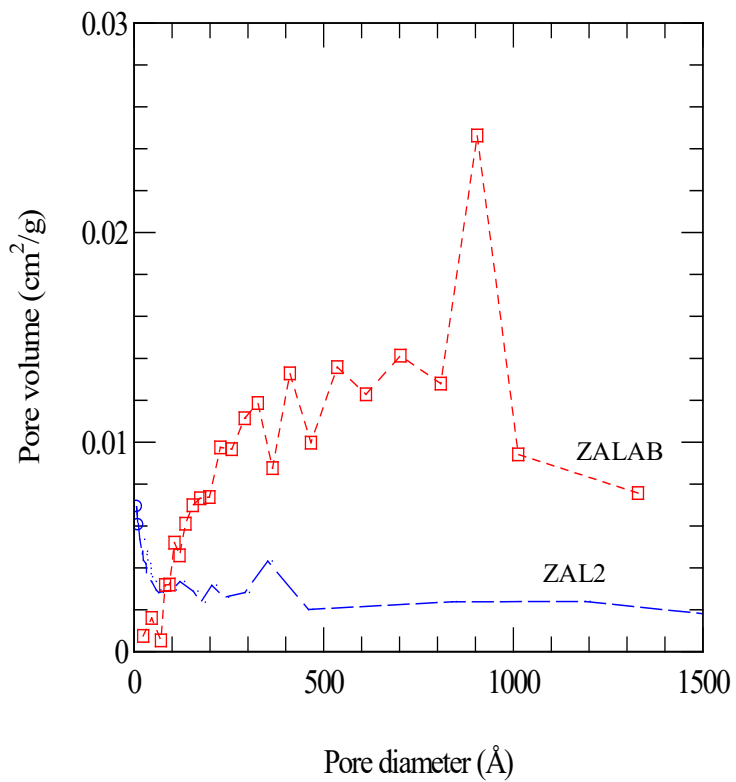

Figure 9 BJH pore size distribution for ZAL2 and ZALAB. 
Table 1 Surface area and pore properties of ZAL2 and ZALAB.

\begin{tabular}{ccc}
\hline Sample & BET surface area $\left(\mathbf{m}^{\mathbf{2}} \mathbf{g}^{-1}\right)$ & BJH pore diameter $(\AA)$ \\
ZAL2 & 1.26 & 44.5 \\
ZALAB & 4.25 & 134.9 \\
\hline
\end{tabular}

\section{Surface morphology}

FESEM morphologies of ZAL2 and ZALAB at 50,000× magnification are shown in Figures 10(a) and 10(b), respectively. Images for both materials have different surface morphologies, where the ZAL2 image exhibited agglomerate and compact with nonporous structure, while ZALAB is a bigger particle size compared to ZAL2 with irregular shapes in different sizes. Based on the images, the particles sizes for ZAL2 and ZALAB can be estimated in range $(17.9-89.3 \mu \mathrm{m})$ and $(142.9-303.6 \mu \mathrm{m})$, respectively. The increase in particle size shows that the inclusion of $4-\mathrm{AB}$ has changed the pore texture and host structure of ZAL2 [15].
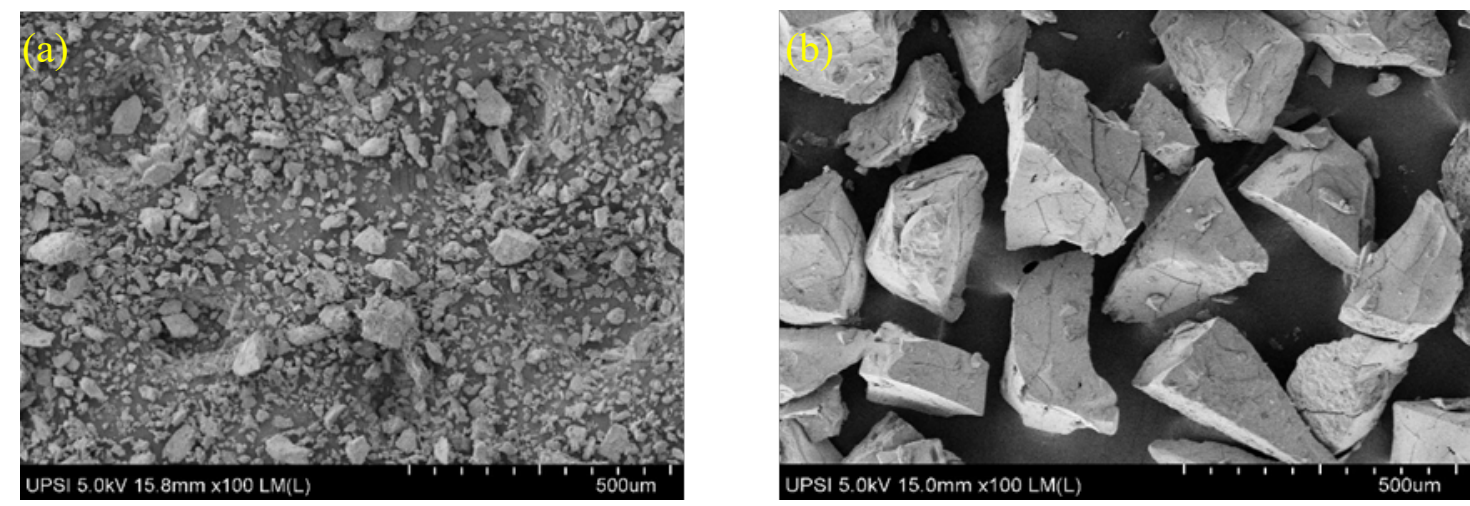

Figure 10 Surface morphology of (a) ZAL2, (b) ZALAB at 50,000 magnification.

\section{Conclusions}

In conclusion, this study has successfully characterized the structural, thermal, and surface properties of ZALAB materials. ZALAB mesoporous material containing 4-AB has a higher thermal stability compared to $4-\mathrm{AB}$ in pure form, demonstrating the potential use of $\mathrm{LDH}$ as thermal protection of organic compounds in sunscreen formulations to enhance pharmaceutical technology for skin care treatments.

\section{Acknowledgements}

This work was financially supported by the Ministry of Higher Education Malaysia (MOHE) under FRGS Grant Code: 2015-0160-101-02. One of authors would like to thank to Sultan Idris Education University for Graduate Research Assistant (GRA). 


\section{References}

[1] ACT Cursino, JEFDC Gardolinski and F Wypych. Intercalation of anionic organic ultraviolet ray absorbers into layered zinc hydroxide nitrate. J. Colloid Interf. Sci. 2010; 347, 49-55.

[2] RL Rietschel, JF Fowler and AA Fisher. Fisher's contact dermatitis. Bc Decker, New York, 2008, p. 88-110.

[3] BS Mackie, LE Mackie and Australas. Reactions with Aminobenzoic acids via diazonium salts open new routes to bio-derived aromatics. J. Dermatol. 1999; 40, 51-3.

[4] CD Hoyo. Layered double hydroxides and human health: An overview. Appl. Clay Sci. 2007; 36, 103-21.

[5] L Perioli, V Ambrogi, B Bertini, M Ricci, M Nocchetti, L Latterini and C Rossi. Anionic clays for sunscreen agent safe use: Photoprotection, photostability and prevention of their skin penetration. Eur. J. Pharm. Biopharm. 2006; 62, 185-93.

[6] Y Feng, D Li, Y Wang, DG Evans and X Duan. Synthesis and characterization of a UV absorbentintercalated Zn-Al layered double hydroxide. Poly Degrad. Stabi. 2006; 91, 789-94.

[7] IT Roto and Mustofa. Zn-Al layered double hydroxide as host material for sunscreen compound of p-aminobenzoic acid. Indo. J. Chem. 2007; 7, 1-4.

[8] INFA Aziz, SH Sarijo, FSM Rajidi, R Yahaya and M Musa. Synthesis and characterization of novel 4-aminobenzoate interleaved with zinc layered hydroxide for potential sunscreen application. $J$. Porous Mater. 2019; 26, 717-22.

[9] Q He, S Yin and T Sato. Synthesis and photochemical properties of zinc-aluminum layered double hydroxide/organic UV ray absorbing molecule/silica nanocomposites. J. Phys. Chem. Solids 2004; 65, 395-402.

[10] JH Choy, SY Kwak, YS Han and BW Kim. New organo-montmorillonite complexes with hydrophobic and hydrophilic functions. Mater. Lett. 1997; 33, 143-7.

[11] L Qin, M Wang, R Zhu, S You, P Zhou and S Wang. The in vitro sustained release profile and antitumor effect of etoposide-layered double hydroxide nanohybrids. Int. J. Nanomedicine 2013; 8, 2053-64.

[12] SH Sarijo, MZ Hussein, AH Yahaya, Z Zainal and MA Yarmo, Synthesis of phenoxyherbicidesintercalated layered double hydroxide nanohybrids and their controlled release property. Curr. Nanosci. 2010; 6, 199-205.

[13] S Li, Y Shen, M Xiao, D Liu, L Fa and K Wu. Intercalation of 2,4-dihydroxybenzophenone-5sulfonate anion into $\mathrm{Zn} / \mathrm{Al}$ layered double hydroxides for UV absorption properties. J. Indus. Eng. Chem. 2014; 20, 1280-4.

[14] MZ Hussein, Z Zainal, AH Yahaya and DWV Foo. Controlled release of a plant growth regulator, alpha-naphthaleneacetate from the lamella of $\mathrm{Zn}$-Al-layered double hydroxide nanocomposite. $J$. Control. Rel. 2002; 82, 417-27.

[15] SMN Mohsin, MZ Hussein, SH Sarijo, S Fakurazi, P Arulselvan and TYY Hin. Synthesis of (cinnamate-zinc layered hydroxide) intercalation compound for sunscreen application. Chem. Cent. J. 2013; 7, 26.

[16] SH Sarijo, A Ahmad, SMN Muhsin and Z Jubri. Synthesis of layered organic-inorganic nanohybrid zinc-aluminium-2-(4-chlorophenoxy)-2-methyl propionic acid with controlled release properties. $J$. Porous Mater. 2019; 26, 41-50.

[17] F Barahuie, MZ Hussein, SA Gani, S Fakurazi and Z Zainal. Synthesis of protocatechuic acidzinc/aluminium-layered double hydroxide nanocomposite as an anticancer nanodelivery system. $J$. Solid State Chem. 2015; 221, 21-31.

[18] E Conterosito, L Palin, D Antonioli, D Viterbo, E Mugnaioli, U Kolb, L Perioli, M Milanesio and V Gianotti. Structural characterisation of complex layered double hydroxides and TGA-GC-MS study on Thermal Response and Carbonate Contamination in Nitrate- and Organic-Exchanged hydrotalcites. Chem. A Eur. J. 2015; 21, 14975-86.

[19] M Ghadiri, W Chrzanowski and R Rohanizadeh. Antibiotic eluting clay mineral (Laponite) for wound healing application: An in vitro study. J. Mater. Sci. Mater. Med. 2014; 25, 2513-26. 
[20] Y Wei, Y Gao, Q Xie, G Zhang and W Fu. Isolation of chlorogenic acid from Flaveria bidentis (L.) Kuntze by CCC and synthesis of chlorogenic acid-intercalated layered double hydroxide. Chromatographia 2011; 73, 97-102.

[21] Y Zhi, Y Li, Q Zhang and $\mathrm{H}$ Wang. ZnO nanoparticles immobilized on flaky layered double hydroxides as photocatalysts with enhanced adsorptivity for removal of acid red G. Langmuir 2010; 26, 15546-53.

[22] Z Jubri, MZ Hussein, AH Yahaya and Z Zainal. The effect of substitution of zinc with aluminium in the brucite-like layers on the physicochemical properties of zinc-aluminium-layered double hydroxide-pamoate nanocomposite. J. Porous Mater. 2012; 19, 45-51.

[23] M Mamat, E Kusrini, AH Yahaya, MZ Hussein and Z Zainal. Intercalation of anthranilate ion into zinc-aluminium-layered double hydroxide. Int. J. Technol. 2013; 4, 73-80. 